\title{
Mehrsprachigkeit, Übersetzung und Nationalliteratur Deutsche Mácha-Übersetzungen im 19. Jahrhundert
}

\section{Astrid Winter}

The paper is based on the fact that the main work of Czech Romanticism, Karel Hynek Mácha’s Máj (1836), was well received by a German speaking and only later by a Czech speaking readership. By means of the German Máj-translations by Siegfried Kapper (1844), Alfred Waldau (1862), Bernard Alois Rambousek (1871) and Karl Müller (1882) the questions are discussed why the German translators engaged themselves to such an extent in a work of Czech Romanticism the comprehension of which confronted them with enormous complications due to language contrast and how far their translations depended on the conditions of multilingualism and a territorially determined identity in Bohemia.

German Mácha reception - Máj - translation - translation history - multilingualism bilingualism - Kapper - Waldau - Rambousek - Müller - Bohemia

Der Artikel geht von dem Umstand aus, dass das Hauptwerk der tschechischen Romantik, Karel Hynek Máchas Máj (1836), zunächst bei einer deutschsprachigen und erst später bei einer tschechischsprachigen Leserschaft Anklang fand. Anhand der deutschen Máj-Übersetzungen durch Siegfried Kapper (1844), Alfred Waldau (1862), Bernard Alois Rambousek (1871) und Karl Müller (1882) wird die Frage untersucht, warum deutsche Übersetzer sich derart in der Übertragung eines Werks der tschechischen Romantik engagierten, dessen Verständnis sie mit enormen sprachkontrastiv-bedingten Problemen konfrontierte, und inwieweit ihre Textübertragungen den Voraussetzungen der Mehrsprachigkeit und einer territorial bestimmten Identitätsbildung in Böhmen unterlagen.

Deutsche Mácha-Rezeption - Máj - Übersetzung - Übersetzungsgeschichte Mehrsprachigkeit - Bilingualismus - Kapper - Waldau - Rambousek - Müller - Böhmen

\section{Die Ausgangslage ${ }^{1}$}

1836 schuf der Dichter Karel Hynek Mácha (1810-1836) mit der Versdichtung Máj (Mai) das bedeutendste Werk der tschechischen Romantik und etablierte damit im Prozess der nationalen Wiedergeburt das Tschechische endgültig als Literatursprache. Heute, über zwei Jahrhunderte nach Mácha, ist Máj das am häufigsten herausgegebene tschechische literarische Werk, vielfach zitiert, interpretiert, illustriert, verfilmt, inszeniert, mystifiziert und teilweise bis zum erotischen Kitsch verfremdet. Angesichts dieser allgemeinen Wertschätzung ist jedoch der schwierige Weg zur Kanonisierung in der tschechischen Literaturgeschichte heutzutage kaum noch gegenwärtig. So forderten Máchas Grenzüberschreitungen und Normbrüche in sprachlich-ästhetischer und, damit verbunden, in sozialer

Dieser Aufsatz ist in veränderter Fassung auch unter Winter (2013) erschienen. 
und politischer Hinsicht im 19. Jh. sehr widersprüchliche Bewertungen durch die tschechische und deutsche Leserschaft heraus. Da Máj von allen Zielsprachen am weitaus häufigsten ins Deutsche übersetzt wurde, möchte dieser Beitrag am Beispiel der Übertragungen des Poems der Frage nachgehen, inwieweit die deutsche Rezeption im 19. Jh. den Voraussetzungen der Mehrsprachigkeit und einer territorial bedingten Identitätsbildung unterlag.

\section{Die unterschiedliche deutsche und tschechische Rezeptionsgeschichte Máchas}

Ignaz Mácha begann seinen dichterischen Weg - angeregt durch deutsche Rezitationsübungen bei Alois Klar an der Prager Karl-Ferdinands-Universität ${ }^{1}$ zunächst mit deutschsprachigen Gedichten (vgl. Eisner 1945; 1956; Králík 1961; 1962), ging dann - von Josef Jungmann für die tschechische nationale Idee begeistert - ab 1831 als Karel Hynek Mácha (vgl. Králík 1961: 393) zum Tschechischen als Dichtungssprache über und verarbeitete schließlich in Máj verschiedenste Anregungen zu einem exzeptionellen dichterischen Werk, das jedoch zunächst auf tschechischer Seite keinen Widerhall fand. Seine in den Polemiken der Wiedergeburtszeit befangenen tschechischen Zeitgenossen warfen ihm Fremdartigkeit, Byronismus, Pessimismus und fehlenden Patriotismus vor (vgl. Vašák 2004).

Im Gegensatz dazu wurde Máj schon bald nach der Veröffentlichung 1836 in deutschsprachigen Kreisen sehr positiv aufgenommen. Besonders in dem Leipziger Blatt Unser Planet (1836), dem Österreichischen Morgenblatt (1936), in der Prager Zeitschrift Ost und West (1840, 1841), in der Augsburger Allgemeinen Zeitung (1840) sowie in den Franklschen Sonntagsblättern (1842) würdigten Rezensenten das Genie des Dichters, hoben den sprachlich wirkungsvollen Kontrast zwischen poetischer Naturschilderung und subjektiver Zerrissenheit sowie den Willen zur Schaffung einer Literatursprache hervor:

„Der Charakter der slawischen Muse dünkt uns größtenteils ergreifend, und doch sanft, [...] wie uns auch der größte Teil böhmischer, polnischer und russischer Volksmelodien [...] so innig anspricht. [...] Des Autors reges Gefühl spricht sich besonders in jenen Stellen recht ergreifend aus, wo er das romantisch Schöne der Natur und des Unglücks schildert. " 2

$1 \quad$ Alois Klar (s. a. Weinolt 1835) war Gründer einer Blindenanstalt auf der Prager Kleinseite und als Professor der Klassischen Philologie Nachfolger August Gottlieb Meißners. Begleitend zu seinen Deklamatorien veröffentlichte er 1822 und 1829 zwei Textsammlungen mit Beispielen vorromantischer deutscher Dichtung sowie mit Gedichten seiner Schüler. Dem Kreis um Klar gehörten auch Chmelenský und Čelakovský, die späteren Kritiker Máchas, an (vgl. Fischer 1929: 248f.). Máchas deutsche Gedichte von 1829 (erstmals veröffentlicht 1882) stehen offenbar in direktem Bezug zu Klars Rezitationsübungen (s. a. Josef Mach in Janský 1958: 197).

2 N. N-ky: Einige Worte über Böhmens neueste Nationalliteratur (1836) zit. n. Mácha (2006: 86). -

Brünner Hefte zu Deutsch als Fremdsprache • Jahrgang $6 \bullet$ Nummer 1/2 • 2013 
Implizit sah man in dieser unterstellten „slawischen Romantik“ (Mácha 2006: 96) einen Einheitsgedanken verwirklicht, der den deutschen Dichtern im revolutionären Vormärz als Vorbild dienen konnte. Darüber hinaus könnte man vermuten, dass sich in der Wahrnehmung der formalen Innovation durch den deutschsprachigen Leser auch die bilinguale Prägung Máchas spiegelte, obwohl dieses Werk allein im System der tschechischen Sprache wurzelt. Denn die positiven deutschsprachigen Wertungen erschienen in Periodika, deren Herausgeber sich trotz zunehmender politischer Spannungen um eine ausgewogene Darstellung tschechischer, deutscher und jüdischer Autoren bemühten und vor allem landespatriotische, sog. ,bohemistische Ansichten vertraten. ${ }^{1}$ Auch Mácha selbst kann zwar eine patriotische, aber keine antideutsche Haltung unterstellt werden, und Einiges scheint darauf hinzudeuten, dass er an einer deutschsprachigen Leserschaft interessiert war. ${ }^{2}$

Erst Jahrzehnte nach Máchas Tod wurde Máj durch die Generation der Májovci (nun gerade aufgrund der ästhetischen Innovation) als Werk der tschechischen Nationalliteratur kanonisiert. Mit der Verehrung des Verspoems begann sich ein regelrechter Kult um den Dichter und seine Grabstätte in Leitmeritz/Litoměřice zu entwickeln. ${ }^{3}$

Während jedoch der tschechische Leser ein Vierteljahrhundert auf die Neuauflage des Máj im Rahmen der ersten Werkausgabe (1861) warten musste, nachdem die von Mácha 1836 im Selbstverlag mit einer Auflage von 600 Exemplaren vertriebene Ausgabe schon bald vergriffen war und nur wenige handschriftliche Kopien kursierten, stand dem deutschen Leser bereits 1844 die erste vollständige Übersetzung in dem Prager Almanach Libussa zur Verfügung. Daran schloss sich eine lange Folge deutscher Übersetzungsversuche an, die auch im 21. Jh. nicht abreißt: Die jüngste Version legte der tschechisch-österreichische AltphilologieStudent, Schriftsteller und Aktionskünstler Ondřej Cikán (vgl. Mácha 2012) in ungewöhnlicher medialer Inszenierung vor. ${ }^{4}$

Zu den ersten Verfassern der deutschen Mácha-Würdigungen zählten auch tschechische Autoren wie Sabina, Ohéral und J. J. Kolár (vgl. Krčma 1932a: 407). Zu weiteren Rezeptionszeugnissen s. Vašák (2004), zur Máj-Rezension in Unser Planet, die eine Teilübersetzung des ersten Intermezzos enthielt, s. a. Vašák (1978).

1 Von besonderer Bedeutung war die von Rudolf Glaser zwischen 1837 und 1848 herausgegebene Zeitschrift Ost und West, in der Beiträge u. a. von Gutzkow, Ebert, Bach, Horn, Hartmann, Kapper, Herloßsohn, Frankl, Sabina, Vinařický und J. J. Kolár veröffentlicht wurden. Der Landespatriot Glaser vertrat die Position, dass Böhmen eine Brücke zwischen Ost und West, zwischen Slaven und Deutschen bilden könne und verfolgte, angeregt durch Goethes Begriff der, Weltliteratur', mit seinen Beiträgen und Übersetzungen das Konzept einer slavisch-deutschen Wechselseitigkeit, obwohl seine Vermittlungsabsichten weniger im Inland als vielmehr in Deutschland gewürdigt wurden (vgl. Hofmann 1957: 27-42). Vgl. z. B. die dichterische Ausformulierung dieser Überzeugungen bei Robert Zimmermann (1844): „Čechenblut und deutsches Blut, / Sollt Euch friedlich minnen, / Kleinen Neid und bösen Muth / schlagt Euch aus den Sinnen!“ (Peřina 1996: 117).

2 Die offenbar von Mácha selbst angefertigte deutsche Fassung des Gedichts Rozprostřela chladná noc (Die kühle Nacht) sowie eine Teilübersetzung von Cizinec im Nachlass des Dichters haben zu der Vermutung geführt, dass Mácha eine deutsche oder zweisprachige Publikation geplant haben könnte (vgl. Mácha 1959: 340, 470; Peřina 1996: 107f.).

3 Zum Mácha-Kult s. u. a. Krčma (1932b) und Tureček (2010).

4 Vgl. URL <http://www.youtube.com/watch?v=BPRub3X4aZE> (1.4.2013). 
In der Dialektik der tschechischen Aufnahme im 19. Jh., die von der Ablehnung eines fremd und unpatriotisch wirkenden Dichters bis zur mystifizierenden Überhöhung einer nationalen Kultfigur reichte, spiegeln sich die komplizierten soziokulturellen Voraussetzungen der Rezeption. Das aus einem bilingualen Umfeld hervorgegangene tschechische Werk konzeptualisiert damit auf unterschiedlichsten Ebenen den kulturellen Raum der böhmischen Länder bzw. Prags. Die wechselhafte Rezeptionsgeschichte kann einerseits als Beispiel für die zunehmend sprachlich definierte Grenzziehung im konfliktreichen Zusammenleben von Deutschen und Tschechen dienen, zugleich aber auch als Beleg für die kulturelle Symbiose und ein gegen nationalistische Tendenzen gerichtetes Streben nach Integration und Transfer.

Angesichts der Kanonisierungsverzögerung stellt sich die Frage, ob die Wahrnehmung spezifischer Eigenschaften des Werkes, die zur Ablehnung auf der einen Seite geführt haben, vielleicht gerade die Anerkennung auf der anderen bewirkt haben könnten. Warum nahmen sich die deutschen Übersetzer eines Werkes an, das seiner Übertragung hohe sprachliche Hürden entgegensetzte?

\section{Die Übersetzer - ihre Motive und Strategien}

Trotz der großen Anzahl an Übersetzungsversuchen ist ihre wissenschaftliche Untersuchung eher ein Randgebiet der Mácha-Forschung geblieben. Die Gründe dafür liegen zweifellos einerseits in der diskontinuierlichen Etablierung des Werkes im Rahmen der tschechischen Literaturgeschichtsschreibung, andererseits in der unvollständigen Überlieferung der Übersetzungen wie auch in den politisch motivierten, sprachnationalistischen Abgrenzungstendenzen in den böhmischen Ländern, die die Vermittlung tschechischer Literatur im deutschen Sprachgebiet erheblich erschwerten. ${ }^{1}$

\subsection{Siegfried Kapper (1821-1879)}

1844 veröffentlichte der junge Medizinstudent Siegfried Kapper die erste vollständige Übersetzung, die allgemein wohlwollend bewertet wurde (vgl. Donath 1937: 329) und der Theodor von Grünwald im Vorwort bescheinigte, „den Geist des Originales in den Garten deutscher Sprache mit glücklichem Erfolge verpflanzt“" (Mácha 1844: 100) zu haben. Als Dichter und Übersetzer jüdischer Herkunft war Kapper einer der wichtigsten Vermittler tschechischer Literatur in der Vormärz-Zeit, bemühte sich aber vergeblich um die Anerkennung seiner eigenen tschechischen Dichtungen und litt als glühender Anhänger des Jungen Böhmen (Mladá Čechie) besonders unter den antisemitischen Ressentiments der tschechischen Patrioten (vgl. Donath 1923: Mit einzelnen Übersetzungen haben sich Donath (1937), Polák (1940), Schamschula (1983),
Nezdařil (1985), Jähnichen (1967; 1972; 2000) und Winter (2010; 2011) befasst. 
12-15; 1934: 329f.), sodass er nach 1848 nur noch deutsch dichtete. Für ihn war Mácha ein „urthümlicher, riesenkräftiger, selbstständiger Genius“ der jungen „böhmischen Nationalliteratur“ (Kapper 1842: 313). „Mácha hatte die Kraft, der Literatur seiner Nazion [sic] eine eigene Richtung zu geben - der Gründer einer Schule zu werden, - was bisher noch keiner der böhmischen Poeten und Literaten vermocht!“ (ebd.). Zugleich warf er den tschechischen Zeitgenossen vor, das Erbe Máchas nicht zu würdigen: „Sind Euch die Reliquien eines vaterländischen Geistes, wie Mácha - sind Euch die Reliquien Eures vielleicht größten Dichters so gering, daß Ihr sie vermodern, verwerfen, verkramen, unter altem, unbrauchbarem Makulaturpapier vertrödeln lasset?“ (Kapper 1842: 314).

Im Überschwang seiner Nachdichtung erlaubte sich der noch unerfahrene Übersetzer allerdings einige Freiheiten. ${ }^{1}$ So vereinheitlichte er den wechselnden Rhythmus des Originals zu einem regelmäßigen Jambus, der im Deutschen nicht charakteristisch ist, und verzichtete häufig sogar auf den Kontrast zu den ikonisch eingesetzten daktylischen Versen, die, wie z. B. im zweiten Intermezzo, mit metrischen Mitteln ein Fallen signalisieren:

Mácha: „[...] nepohnutým kolem zvučí: / ,Vůdce zhynul! - vůdce zhynul!““ (Mácha 2002: 36, 715-716). ${ }^{2}$

Kapper: ,[...] Hallt lauter es im Chore toll und wild / ,Der Hauptmann ist gefallen - ist gefallen!'“ (Mácha 1844: 121).

Kapper erweiterte, glättete und rhetorisierte die Vorlage:

Mácha: „[...] a slunce jasná světů jiných / bloudila blankytnými pásky, / planoucí tam co slzy lásky" (Mácha 2002: 13, 13-15).

Kapper: „[...] Indeß der Sonne Strahlen - Glutguirlanden - / Gen andre Welten irrend, hier verschwanden, / Um dort zu glüh'n, gleich heißer Liebe Zähren“"(Mácha 1844: 101).

Daneben erzielte er aber auch eine später kaum in dieser Form realisierte Umsetzung der Lautinstrumentierung des Originals: in den Eingangsversen durch Alliterationen (traut - treu, Liebe - lud), Wiederholung von Vokalen und Konsonanten aus den bedeutungstragenden Lexemen Liebe, Rose und Mai (i/ü, o, a; l, r, m), durch Polyptota (Liebe, Liebes-) und einen fließenden, nicht monotonen Rhythmus. Kapper erreichte dies um den Preis größerer Abweichungen vom Original, etwa

$1 \quad$ Die Defizite der Kapperschen Verse sind u. a. bemängelt worden von Donath (1937: 331-333) und Polák (1940: 216-218). Jähnichen verweist sowohl auf Abweichungen und Interpretationen als auch auf gelungene ästhetische Äquivalente Kappers, schränkt seine frühere sehr positive Einschätzung der Übersetzung (vgl. Jähnichen 1967: 239-264) aber später ein (vgl. Jähnichen 2000: 137-139).

2 Im Folgenden sind die Zitate aus dem Original der jüngsten Ausgabe entnommen (Mácha 2002). Die der Seitenzahl folgende Zahl entspricht der Versnummerierung. Einzelne Wörter werden in Kursivschrift, Verse in Anführungszeichen zitiert. 
durch Erweiterungen der Verse um mehrere Ikten, Kreuzreimschema (aBa ${ }^{\circ} \mathrm{BCdCd}$ statt abbacddc), unbetonte Kadenzen und fehlende Zäsuren in der Versmitte:

Mácha: „Byl pozdní večer - první máj - / večerní máj - byl lásky čas. / Hrdliččin zval ku lásce hlas, / kde borový zaváněl háj. / O lásce šeptal tichý mech; / květoucí strom lhal lásky žel, / svou lásku slavík růži pěl, / růžinu jevil vonný vzdech“ (Mácha 2002: 13, 1-4). Kapper: „Spät Abend - erster Mai - ein Abendmai. / Es war die Zeit, um liebend zu durchirren / Den duft'gen Kieferhain, wo traut und treu / Zur Liebe lud der Turteltäubchen Girren. / Von Liebe flüstern rings die weichen Moose, / Der Liebe Wehe log der Blüthenbaum, / Die Nachtigall sang Liebesklag' der Rose, / Verduftend still, ein glüher Liebestraum“ (Mácha 1844: 100).

Kapper deutete und konkretisierte die offene, anspielungsreiche Metaphorik des tschechischen Textes im Sinne der deutschen Romantik. Wenn er z. B. - bisher in der Literatur unkommentiert - „lesní to trouba v noční čas / uvádí hudbu jemnou“ (Mácha 2002: 22, 271f.) mit „Es ist in ferner Waldeinsamkeit / Des Waldhorns Liedermacht" (Mácha 1844: 107) wiedergab, verwies er auf einen Schlüsselbegriff der deutschen Romantik, den Ludwig Tieck 1797 erstmals verwendet hatte. So schuf Kapper mit seiner freien Nachdichtung einen rhetorisch-pathetischen Mácha im Geist des Vormärz.

\subsection{Alfred Waldau (d. i. Josef Jarosch, 1837-1882)}

Unter dem Eindruck der Mácha-Verehrung durch die Májovci einerseits und angesichts der antitschechischen Polemik in der deutschen Presse andererseits gab der aus Mittelböhmen stammende deutschsprachige Jurist und Offizier Josef Jarosch unter dem Pseudonym Alfred Waldau 1862 eine weitere Übersetzung des Máj heraus (Mácha 1862: 42-86). Ihr waren 1858 der tschechische Almanach Máj und 1861 die erste Werkausgabe Máchas vorangegangen. In den 1860er Jahren kam die Herausgabe deutschsprachiger Anthologien tschechischer Dichtung fast zum Erliegen, weil sich ihre Verleger dem Vorwurf der Parteinahme für die tschechische Seite ausgesetzt sahen (vgl. Kaiser und Michler 2002: 194f.). Insofern erhalten die Vermittlungsbemühungen Waldaus, der mit Jan Neruda befreundet war und die deutschsprachigen Leser auch mit böhmischen Tänzen, Märchen und Volksliedern sowie mit den Werken Háleks, Havlíčeks und Hankas bekannt machte, besonderes Gewicht.

In einer von Sabina ausgehenden biographischen Studie im Vorwort seiner Übersetzung würdigte Waldau Mácha als einen der wichtigsten Dichter der „neuböhmischen“ Literatur. Zugleich warf er den deutschen Lesern vor, dass sie sich zwar für die Lieder der „Eskimos“ und „Congo-Neger“ interessierten, aber über die Literatur des engsten Nachbarn nur wenig wüssten, obwohl Böhmen eine Brücke zwischen Ost und West bilde (vgl. Mácha 1862: 15f.). Sehr genau registrierte er 
Máchas Nihilismus: „Scharf und kühn blickte er den letzten Folgen der Negation ins Antlitz" (vgl. Mácha 1862: 33).

Obwohl er die sprachliche Wirkung des Textes quasi philologisch analysierte, zeigt seine Übersetzung eine Tendenz zur semantischen Reduktion des Originals, an einigen Stellen zur freien Interpretation, auf die bereits Donath (1937: 334f.) hingewiesen hat.

Mácha: „[...] Vtom lůny zár / jí známou osvítila tvár [...]“ (Mácha 2002: 16, 106).

Waldau: „[...] das fahle Vollmondlicht / Beschien ein fremdes, kaltes Angesicht $[\ldots]$ “ (Mácha 1862: 49).

Außerdem beruht sie zu etwa einem Drittel auf der Vorlage Kappers, so dass diese Filiation dieselben Züge der rhetorisierenden Verserweiterung und rhythmischen Vereinheitlichung durch den merkmallosen Jambus trägt wie ihr Vorläufer. Stellenweise suchte Waldau wie Kapper nach lautmalerischen Äquivalenten von Máchas Klangwiederholungen:

Mácha: „[...] řetězů řinčí hřmot, a pak / u tichu vše umírá“ (Mácha 2002: 21, 262-264). Waldau: „[...] Wildrasselnd fiel die Eisenkette nieder, / und alles war wie ausgestorben wieder" (Mácha 1862: 57).

Auch wenn die Übersetzung Waldaus gravierende Schwächen zeigt, erhält sie durch die in ihrer Ausgabe enthaltenen 59 weiteren Gedichte Máchas besondere Bedeutung. Diese Gedichtsammlung sollte aber bis zum Erscheinen der gesammelten Werke (Mácha 2000) die wichtigste deutsche Mácha-Ausgabe bilden und konnte sogar zur Rekonstruktion des fragmentarisch erhaltenen Sonetts Jest pěvcưv osud světem putovati beitragen (vgl. Mácha 2002: 352f.). Waldau bereicherte damit die MájRezeption um eine Variante, die im Sinne der Májovci die formale und inhaltliche Unangepasstheit Máchas betonte, um in der konfliktreichen österreichischen Verfassungsperiode die weltliterarische Geltung der tschechischen Literatur unter Beweis zu stellen.

\subsection{Bernard Alois Rambousek (1847-1880)}

Erst 2009 wurde die noch 1886 von Jakub Arbes (1941:372) erwähnte und schon von Polák (1940: 220) verloren geglaubte Máj-Übersetzung Bernard Alois Rambouseks aus dem Jahr 1871 im literarischen Nachlass Antonín Pikharts (1861-1909), des bedeutenden Übersetzers aus dem Spanischen, durch Zufall wiederentdeckt (vgl. Uličný 2010a; 2010b: 9-23). Es handelt sich um eine Abschrift Pikharts von 1883, in der dieser zu Beginn erklärt, die Rechte einer ungenannten Besitzerin des Manuskripts nicht verletzen zu wollen. Anlass und Zweck der Übersetzung 
sind unklar. Dass sie zur Veröffentlichung bestimmt war, erscheint angesichts der allgemein erschwerten Publikationsmöglichkeiten und der geringen literarischen Erfahrungen des früh verstorbenen tschechischen Armenarztes Rambousek eher unwahrscheinlich. Möglicherweise handelte es sich bei der unbekannten Besitzerin um die Adressatin einer privaten Liebesgabe, wahrscheinlich um Marie Grubner, die offenbar aus einer deutschen Familie stammende Frau Rambouseks. ${ }^{1}$

Obwohl einige Verse fehlen (evtl. ein Versehen des Abschreibers), zeigt der Übersetzer ${ }^{2}$ ein starkes Bemühen, das Original in der rhythmischen und syntaktischen Struktur wiederzugeben.

Rambousek: „Spät Abend ist's - Der erste Mai - / Ein Abendmai - Der Liebe Zeit; / Zur Lieb' die Turteltaube schreit / Im duft'gen Fichtenwald herbei. / Im Moos ein leiser Liebeschall; / Der Blüthenbaum verliebt ist auch, / Von Liebe spricht der Rose Hauch, / Zu ihr aus Lieb’ singt Nachtigall“ (Mácha und Rambousek 2010: 45).

In den Eingangsversen übertrug Rambousek nicht nur die Zäsuren sowie die Inversionen (lásky čas - der Liebe Zeit), sondern auch die umschließenden Reime (abbacddc) mit betonter Kadenz nach Máchas Vorbild, stellenweise sogar in lautlicher Übereinstimmung (máj- háj, Mai - herbei; mech-vzdech, auch-Hauch). Dabei ahmte er zudem noch Máchas akzentuierte Monosyllaben in Reimposition nach.

Das voll realisierte Versschema des vierfüßigen Jambus $(v-v-v-v-)$ stimmt mit der natürlichen Akzentuierung überein, wirkt aber - besonders durch das im Deutschen nicht notwendige einsilbige Auftaktwort - monoton und durch die zahlreichen poetischen Kürzen (ist's, Lieb', duft'gen) rhythmisch stockend. Daneben versuchte Rambousek zwar die Lautwiederholungen der Schlüssellexeme durch Vokalhäufung und Wiederholungen bzw. Alliterationen zu berücksichtigen, vermochte aber dem lautmalerischen Original nicht zu entsprechen. In den meist reinen Reimen, die gemäß dem Vorbild oft einsilbig im männlichen Versschluss den Jambus noch verstärken und das Máchasche Reimschema überwiegend realisieren, ließ der Übersetzer - wohl unbewusst entsprechend der regionalsprachlichen Varianten der Umlautartikulation - auch [y]-[i]-, [œ]-[e]- und [c]-[e]-Gleichklänge (Gründe - Winde, Glück - Geschick, Höh - Weh, Tön' - stehn, Händ - gelehnt) zu, die bei Kapper nicht auftraten. Da die Verslänge meistens dem Original entspricht, bewirken die in der silbenreicheren deutschen Sprache notwendigen Umstellungen oft syntaktische Fehler.

Im Bemühen um formale Parallelität geht die semantische Äquivalenz verloren; dies wirkt zuweilen unfreiwillig komisch: Wenn etwa die Turteltaube nicht zur Liebe ruft, sondern „schreit“ oder „Der Blüthenbaum verliebt ist“ statt gelogen zu haben

\footnotetext{
1 Vgl. die Todesanzeige im Stadtarchiv Jablonec in Mácha und Rambousek (2010: 177).

2 Zu dieser Übersetzung s. ausführlicher Winter (2010: 104-115).
} 
(lhal), eine botanische Unsicherheit ohne Not aus Kiefern Fichten macht (borový háj), Synästhesien nicht erfasst, sondern interpretiert werden (sladký zvuk-süße Klage) oder Interferenzen des Tschechischen bzw. regionalsprachliche Varianten einfließen (brát si - sich spielen). Stellenweise konterkarieren unangemessene Diminutive und Attribute die dramatische Situation:

Mácha: „Ach zemi krásnou, zemi milovanou / kolébkou mou i hrob můj, matku mou“ (Mácha 2002: 36, 616f.).

Rambousek: „Das Land grüszt mir, wo meine Wiege stand, / die schöne Erd', mein liebes Mütterlein“" (Mácha und Rambousek 2010: 89).

Auch löst Rambousek die asyndetisch gereihten parallelen Genitivinversionen der Máchaschen Oxymora-Ketten in Präpositionalphrasen auf, was die Wirkung der sich steigernden Negation stark abschwächt:

Mácha: „[...] zbortěné harfy tón, ztrhané strůny zvuk [...]“ (Mácha 2002: 42, 804).

Rambousek: „,...] ein Ton von Harfen mit gesprungenen Saiten [...]“ (Mácha und Rambousek 2010: 101).

Interessanterweise hat sich Rambousek von Kappers oder Waldaus Vorlagen kaum beeinflussen lassen. Sein eingeschränkter Wortschatz im Deutschen führte, besonders in phraseologischer Hinsicht, zu stilistischer Unsicherheit. Trotz dieser Schwächen ist die außerordentliche Leistung Rambouseks zu würdigen. Er scheint die Intention verfolgt zu haben, eine möglichst formal getreue Wiedergabe des Ausgangstextes zu erreichen, um aus Achtung gegenüber dem Autor den verinnerlichten, fast könnte man sagen ,sakralisierten' Text in einer anderen Sprache, die er ebenfalls sehr gut beherrschte, die aber dennoch nicht seine Muttersprache war, originalgetreu wiederzugeben und dessen Schönheit zu vermitteln. Dabei stellte er den Inhalt häufig unfreiwillig - in den Dienst der Form.

\subsection{Karl Müller (1837-1892)}

In zeitliche Nähe zur Übersetzung Rambouseks rückt eine weitere Neuübersetzung des Werks von 1882, die der Freidenker und demokratische Journalist Karl Müller gleich zweimal anfertigte, da seine erste Version verloren gegangen war. Er stammte aus einer deutschen Familie und redigierte in Pilsen und Prag verschiedene Zeitschriften. Als sich in der Taaffe-Ära aufgrund politischer Zugeständnisse (1880 Sprachenverordnung, 1882 Universitätsteilung, 1883 tschechische Mehrheit im böhmischen Landtag) die antitschechische Stimmung in der deutschen Presse weiter verstärkte (vgl. Křen 1990: 212-237), setzte er sich mit seinen Übersetzungen sehr engagiert für die Vermittlung und Anerkennung der tschechischen Literatur in Österreich und Deutschland ein (vgl. Jähnichen 1972: 84-102). Er erlangte 
damit jedoch nur regionale Aufmerksamkeit. Nicht zuletzt aufgrund verschärfter Zensur- und Publikationsbedingungen konnten nur wenige seiner zahlreichen Übersetzungsprojekte, mit denen er vor allem ein Bild der zeitgenössischen Literatur (Čech, Vrchlický, Neruda u. a.) vermitteln wollte, in Buchform erscheinen. Viele Übertragungen blieben in Zeitschriften verstreut.

Im Unterschied zu Kapper und Waldau zeigt Müllers Übersetzung hinsichtlich der Verslänge, des Reimschemas und Metrums eine größere Nähe zum Original, wie schon die ersten Verse der Máj-Übersetzung (Mácha 1882: 15) zeigen: „Spät Abend war's - am ersten Mai - / Maiabend war's - der Liebe Zeit“ ( $v-v-v-v-$ I $-\mathrm{v} v-\mathrm{v}-\mathrm{v}-$ ). Müllers Version ist sprachlich-stilistisch gewandter als Rambouseks stellenweise unbeholfener Versuch, weist aber auch semantische Abweichungen auf, die - wie schon Donath (1937: 335f.) anmerkte - zu freien Paraphrasen führen können: Aus Máchas „Jezero hladké v křovích stinných“ (Mácha 2002: 13, 9) wird bei Müller „Des glatten See’s krystall'ner Bronnen“ (Mácha 1882: 15). Einige Verse wie z. B. „Der Körper starr - die Zung' ohn' Laut, / Das Auge starr in’s Leere schaut“ (Macha 1882: 29) werden hinzugefügt, andere Verse werden schlicht unterschlagen.

Auch wenn Müller zweifellos politische Absichten verfolgte, nannte er im Vorwort andere Motive: Er wolle den deutschen Leser - trotz der bereits vorliegenden Übersetzungen Kappers und Waldaus - mit Mácha bekannt machen, weil die Gedichte „für immer“ seine „liebste Lektüre“ gewesen seien und diese Liebe zu Mácha schon in seiner Gymnasialzeit in Leitmeritz geweckt worden sei, als er Fiktion oder nicht - getreu dem Mácha-Kult wöchentlich an das Grab des Dichters gepilgert sei (vgl. Mácha 1882: 5f.). In einer captatio benevolentiae entschuldigt er sich dafür, das Original nicht erreichen zu können und begründet dieses Defizit mit dem Hinweis auf die Schwierigkeit, „mit welcher jeder Uebersetzer aus dem Böhmischen in’s Deutsche zu kämpfen“ (Mácha 1882: 7) habe.

\section{Das ästhetische Potential des Ausgangstextes}

Um zu einer Einschätzung der Motive gelangen zu können, gilt es, den Ursprungstext in seiner Neuartigkeit mit den Schwierigkeiten, die er der Übertragung ins Deutsche entgegensetzte, ${ }^{1}$ die ihn aber auch für eine Rezeption interessant gemacht haben könnten, vor dem Rezeptionshorizont der zeitbedingten übersetzerischen und literarischen Normen kurz zu charakterisieren.

Der 824 Verse umfassenden Versdichtung ${ }^{2}$ liegt zwar eine auf historischen Ereignissen beruhende Fabel zugrunde. Doch wird der Sujetaufbau in vier

$1 \quad$ Bereits der anonyme Artikel Übersicht der neuesten böhmischen belletristischen Literatur in der Leipziger Zeitung Unser Planet vom 21. 7. 1836 beurteilt Máchas Máj als „unnachahmlich und unübersetzlich" (Mácha 2006: 100).

2 Stellvertretend für die beträchtliche Anzahl von Máj-Untersuchungen sei hier auf die zusammenfassende Studie von Dušan Prokop (2010) hingewiesen. 
Gesängen und zwei Intermezzi nicht durch die drastische Geschichte des am Ende hingerichteten Räuberhauptmanns bestimmt, der im eigenhändig erschlagenen Verführer seiner Geliebten im Nachhinein den eigenen Vater erkennt, sondern durch den Gegensatz zwischen dem flüchtigen Menschenleben und der Zyklizität der Natur, wie Mácha (2002: 321) selbst erläuterte. Für die bestimmenden Themen wie existentielle Vereinzelung und menschliche Vergänglichkeit, vergebliche Sinnsuche und unerfüllte Liebe sowie für die Figur des Ausgestoßenen sind in der Forschung die Werke Byrons, Mickiewiczs, Goethes, Schillers und Novalis' als literarische Intertexte genannt worden, obwohl diese Quellen nur teilweise durch Exzerpte in Máchas literarischen Skizzenbüchern belegt werden können. Das wiederkehrende Rosenmotiv geht offenbar auf einen direkten Impuls durch das epische Märchengedicht Die bezauberte Rose von Ernst Schulze (1818) zurück (vgl. Mácha 1972: 35, 70, 392). ${ }^{1}$ Trotz dieser Bezüge bleibt das Werk Máchas in der sprachlichen Virtuosität, besonders in der Verschränkung der lautlichen und semantischen Ebenen singulär und war dadurch eine Herausforderung für jeden Übersetzer, gerade weil die zeitgenössische übersetzerische Norm im Wesentlichen unbedingte Werktreue anstrebte.

Wiederholungsfiguren, Lautinstrumentierung, die semantische Dimension einsilbiger Reimwörter (čas - blas, stín - klín) und eine besondere Musikalität der Verse erzeugen ein dichtes Geflecht von Bedeutungsanklängen, das eine suggestive Wirkung ausübt. ${ }^{2}$ Poetische Bedeutungsoffenheit wird durch Adjektivierungen des Bezugsworts (večerní máj statt ,májový večer) und Substantivierungen des Epithetons erzeugt (šero hor statt šeré hory'). Wie schon in Máchas deutschen Gedichten gehören Inversionen des Genitivattributs - z. B. lásky čas; lásky žel; „Ouplné lůny krásná tvár““ (Mácha 2002: 13, 20) - und Hyperbata - z. B. „Hrdliččin zval ku lásce hlas“" (Mácha 2002: 13, 3); „strašný lesů pán“ (Mácha 2002: 20, 205) - zu den Charakteristika des Textes. Hinzu kommen Chiasmen - z. B. „tak bledě jasná, jasně bledá" (Mácha 2002: 13, 21) - und Stellungsfiguren, kombiniert mit Lautwiederholungen, wie z. B. „Dál blyštil bledý dvorů stín“ (Mácha 2002: 13, 26). Anthropomorphisierungen (mech šeptal; strom lhal; jezero zvučelo) und Synästhesien rufen in den sinnlichen Naturvorgängen die Zeichenfunktion der Welt wach und legen den Einfluss mystischer Schriften sowie barocker Dichtung nahe (vgl. Tschižewskij 1972; Mukařovský 1948: 212-215).

1 Auch wenn Schulze nicht zu den hervorragendsten Vertretern der deutschen Romantik zählte, lässt Přibil (2009: 21) diese genetische Beziehung aus nicht ganz nachvollziehbaren Gründen beiseite. Allgemein zum Einfluss durch die deutsche Literatur s. Striedter (1963) und Grebenícková (2010).

2 Geminationen (bliž a blíž, níž a níž), Polyptota (vlna za vlnou, bříza k boru, k bříze bor), Alliterationen (lhal lásky žel, vonný vzdech), Anaphern, Parallelismen, stabreimartige Lautwiederholungen, Wiederholungen der offenen, dunklen Vokalphoneme der Schlüssel-Lexeme láska, máj und růže (also /a:/, /u:/ bzw. /ou/) - im Kontrast zum engen hohen Vokal /i:/ (slavík) - mit den dazugehörigen Konsonanten (weichen Labialen und Liquiden $/ \mathrm{b} /, / \mathrm{p} /, / 1 /, / \mathrm{m} /$ ) und den ahnungsvolles Flüstern suggerierenden Sibilanten $(/ \mathrm{s} /,|\mathrm{z} /,| \mathrm{c} /,|c ̌ /,| \check{s} /, \mid \check{z} /)$. - Mukařovský hat Máj in zahlreichen Untersuchungen analysiert, vgl. hierzu exemplarisch Mukařovský (1948). 
Den optimistischen Patriotismus seiner Zeitgenossen beantwortete Mácha mit Nihilismus („Nikdy - nikde - žádný cíl“ [Mácha 2002: 18, 151], „myšlenka myšlenkou umírá" [Mácha 2002: 18, 170]), realisiert in Kontrasten, Aposiopesen und antinomischen Parallelismen sich steigernder Oxymora: „zašlého věku děj, umřelé hvězdy svit / zašlé bludice pout', mrtvé milenky cit“" (Mácha 2002: 37, 665f.). Dadurch rief er Widerspruch bei den tschechischen und Zustimmung bei den ersten deutschen Lesern seiner Versdichtung hervor.

Lexikalisch entfernte sich Mácha in Máj, dessen Wortschatz überschaubar ist, von den Normen, die bekanntlich Josef Jungmann als Erneuerer der tschechischen Sprache und Verfasser des deutsch-tschechischen Wörterbuchs z. B. in der Wortbildung von Neologismen kodifizierte. ${ }^{1}$ Unter den Vorzeichen eines noch unsicheren Standards griff Mácha - in durchaus innovativer Weise - auch auf das gesprochene Tschechisch zurück, wobei dialektale Einflüsse fast vollkommen fehlen. Viele Momente verweisen auf seine vorangegangenen deutschen Gedichte, z. B. Motive wie das Nichts (nic) in dem Gedicht O Muse. Ebenso ist der allgemeine Einfluss der deutschen Sprache nachzuweisen (etwa in der Verwendung von Lehnübersetzungen wie truchlorouškaTrauerflor, jednozvučně - eintönig), ${ }^{2}$ die in seiner privaten Korrespondenz allerdings wesentlich deutlichere Spuren hinterlassen hat (vgl. Berger 2000: 123f.) und in den Tagebüchern sogar in Fällen von Codeswitching in Erscheinung tritt (vgl. Mácha 2008: 40-58). Viele der in Máj verwendeten Komposita sind Neologismen Máchas und nicht als solche im Jungmannschen Wörterbuch verzeichnet (vgl. Flajšhans 1937: 193f.).

Dem in der Wiedergeburtszeit gern verwendeten fünffüßigen Trochäus setzte Mácha in virtuoser Weise einen durch den Jambus bestimmten Rhythmus entgegen (739 jambische, 63 trochäische, 22 unregelmäßige Verse), den er mit großer Flexibilität in meist vierfüßigen Versen mit Zäsur, aber auch drei- und siebenfüßigen Versen variabel realisierte (Jakobson 1995). Oft alternieren lange und kurze Versmaße, z. B. vierfüßige Jamben mit Alexandrinern. Die semantisierte polymetrische Gestaltung bezieht Anregungen aus dichterischen Dramen wie etwa Goethes Faust (vgl. Červenka 1989: 421) und erzeugt eine typisch romantisch balladeske Wirkung. Den im Tschechischen bestehenden Konflikt zwischen steigendem Metrum und Anfangsbetonung hat Mácha nicht - wie noch Antonín Puchmajer (1833) - durch monotonen einsilbigen Versbeginn ausgeglichen. Stattdessen verstärkte er ihn sogar durch häufige rhythmische Abweichungen, z. B. durch dreisilbige Wörter in schwacher metrischer Position, ohne dabei jedoch die

Zu Lebzeiten standen Mácha bereits zwei Bände des Jungmannschen Wörterbuchs sowie Dobrovskýs Grammatik zur Verfügung (vgl. Flajšhans 1937: 183, 185).

2 Über mögliche sprachpuristische Bestrebungen Máchas und die Bewertung der Germanismen im Vergleich zum zeitgenössischen Standard sind zahlreiche Kontroversen u. a. von Flajšhans, Havránek und Králík geführt worden. Tatsache bleibt jedoch, dass sich die deutsche Sozialisation Máchas in seiner dichterischen Sprache weiterhin niederschlägt. 
metrische Struktur zu zerstören: „,večerní máj - byl lásky čas“ ( $-\mathrm{v} v-\mathrm{v}-\mathrm{v}-)$, „kde borový zaváněl háj" ( $\mathrm{v}-\mathrm{v} \mathrm{v}-\mathrm{v} v-$ ) (Mácha 2002: 13, 2, 4). So musste dieser bewusst flexibel realisierte Jambus in seiner Zeit als außerordentliche Innovation empfunden werden. Da Mácha bei seinen metrischen Abweichungen offenbar auch Anregungen aus der deutschen Prosodie bezog - etwa mit der Schwächung der Betonung von Präpositionen und Präfixen in schwacher Position beim Jambus (s. a. Ibrahim und Sgallová 2010: 199) - ging diese Wirkung bei der Übertragung in einen regelmäßigen deutschen Jambus - das verbreitetste deutsche Metrum - unweigerlich verloren. Allerdings konnte gerade die Wahrnehmung des Bekannten im innovativ umgeformten Anderen den entscheidenden Impuls für die Übertragungsversuche ins Deutsche geliefert haben.

\section{Fazit: Gegensätzliche Aktualisierungen des Máj im 19. Jahrhundert}

Ergebnis der kursorischen Übersetzungs-Lektüre ist ein Paradoxon: Die sprachliche Einzigartigkeit des Ausgangstextes erschloss sich in der Übersetzung nur solchen Lesern, die eigentlich keine Übertragung benötigten. Die Übersetzer aktualisierten besonders die Vermittlungsabsicht Máchas, fremde, v. a. deutsche Einflüsse zu einem eigenständigen Werk der tschechischen Literatur umformen zu wollen. Da der Versuch, das Gedicht einer einsprachig deutschen Leserschaft zu vermitteln, unweigerlich scheitern musste und immer nur einzelne Aspekte der Musikalität, Lautinstrumentierung, der paradigmatischen sequentiellen Wiederholungen und der semantischen Valenz der Reime sowie der polymetrischen Variationen in eine adäquate poetische Form der Zielsprache überführt wurden, lieferte gerade die offensichtliche Unübersetzbarkeit eines der Hauptmotive für deutsche Übertragungsversuche im 19. Jh.

Dabei spielte für einen Dichter wie Kapper im Vormärz nicht nur die sprachliche Herausforderung eine Rolle, sondern auch eine unterstellte revolutionäre einheitsstiftende Absicht Máchas im Sinne einer slavischen Romantik. Bei Waldau stand in der Zeit der tschechischen Kanonisierung Máchas in den 1860er Jahren der Vermittlungsaspekt im Vordergrund, den Mácha selbst durch Amalgamierung verschiedenster Einflüsse verkörperte. Müller verfolgte eindeutig politische kulturvermittelnde Absichten. In der zunehmend antitschechischen Atmosphäre der 1880er Jahre war die Anerkennung der Leistungsfähigkeit der tschechischen Literatur gleichzeitig ein Argument gegen die proklamierte tschechische Rückständigkeit und für die Legitimität politischer Autonomiebestrebungen. Während die deutschen Übersetzer also neben den sprachlichen Besonderheiten, von denen sie aber z. T. erheblich abwichen, das ,ideologische Potential' aktualisierten und immer noch durch eine landespatriotische Gesinnung beeinflusst blieben, legte der einzige Tscheche, Rambousek, das Schwergewicht allein auf die Wiedergabe der formalen Seite. Bei ihm gaben private Gründe den Ausschlag für den Wunsch, einer deutschsprachigen Leserin ein besonderes poetisches Geschenk zu machen. 
Interessanterweise riefen dieselben Eigenschaften, die von deutschen Rezipienten als Konstituenten der slavischen Romantik besonders gewürdigt wurden, gerade die frühen tschechischen Kritiker Máchas auf den Plan, provozierten ihren Spott und den Vorwurf des mangelnden Patriotismus. So wurde von der tschechischen Kritik bezeichnenderweise anfangs nur das stilistisch stark abweichende, dem Poem vorangestellte Huldigungsgedicht an das tschechische Volk Čechové jsou národ dobrý (vgl. Mácha 2002: 11f.) positiv beurteilt und schon 1836 ausgerechnet von Chmelenský, einem Mácha-Kritiker, in der Vertonung durch Skřivánek herausgegeben (vgl. Vašák 2004: 46). Später hat man es als Konzession gegenüber dem Zeitgeschmack interpretiert. In einigen Werkausgaben und den meisten Übersetzungen fehlt es ganz.

Grundlegend bei allen Übersetzern war eine weitgehende Identifikation mit dem Text, dessen Einzigartigkeit vor dem Hintergrund des tragisch kurzen Lebens Máchas besonders deutlich hervortrat. Da der Ausgangstext selbst Einflüsse aus der deutschen Sprache und Literatur bezog, konnte für den deutschen Übersetzer gerade das Bekannte im Fremden als Impuls dienen, die Schönheit in der eigenen Sprache vermitteln zu wollen - eine ästhetische Herausforderung, die sich zwar dem bilingualen Leser erschloss, die aber allein in der Übersetzung ohne Kenntnis des Ausgangstextes letztlich enttäuschen musste und schon im Original die Ablehnung der tschechischen Leser provozierte.

\section{Literaturverzeichnis}

Arbes, Jakub (1941): Karel Hynek Mácha. Studie literární a povahopisná. Praha, Melantrich.

Berger, Tilman (2000): Anmerkungen zur Sprache von Máchas Briefen. In: Schmid, Herta (Hg.): Kapitel zur Poetik Karel Hynek Máchas. München, Sagner. S. 108-132.

Červenka, Miroslav (1989): Polymetrie Máje. In: Česká literatura 37/1989. S. 413-430.

Donath, Oskar (1923): Židé a židovství v české literatuře 19. století. Brno, Píša.

Donath, Oskar (1934): Siegfried Kapper. In: Jahrbücher der Gesellschaft für Geschichte der Juden in der Čechoslovakischen Republik 6/1934. S. 323-396.

Donath, Oskar (1937): Německé překlady Máchova Máje. In: Novák, Arne (Hg.): K. H. Mácha. Osobnost, dilo, oblas. Praha, Družstevní práce. S. 327-340.

Eisner, Pavel (1945): Na skále. Praha, Pavel Voleský.

Eisner, Pavel (1956): Okusy Ignaze Máchy. Praha, Československý spisovatel.

Fischer, Otokar (1929): K. H. Máchas deutsche Anfänge und der Kreis um Alois Klar. In: Kraus, Ernst / Janka Joseph, (Hg.): Xenia Pragensia. Pragae, Sumptibus Societatis Neophilologorum Societatis Mathematicorum et Physicorum. S. 233-259.

Flajšhans, Václav (1937): Hlas Prahy. Rozbor jazyka Máchova. In: Novák, Arne (Hg.): K. H. Mácha. Osobnost, dílo, oblas. Praha, Družstevní práce. S. 179-214.

Grebeníčková, Růžena (2010): Máchovské studie. Praha, Academia.

Hofmann, Alois (1957): Die Prager Zeitschrift Ost und West. Berlin, Akademie-Verlag. Ibrahim, Robert / Sgallová, Květa (2010): Máchův jamb a prostředky zeslabování konfliktu jazyka a metra. In: Piorecký, Karel (Hg.): Máchovské rezonance. Praha, Ústav pro českou Literaturu AV ČR, Nakl. Akropolis. S. 195-202. 
Jähnichen, Manfred (1967): Zwischen Diffamierung und Widerhall. Berlin, Akademie-Verlag. Jähnichen, Manfred (1972): Der Weg zur Anerkennung. Berlin, Akademie-Verlag.

Jähnichen, Manfred (2000): Zu den Versuchen, Máchas Máj ins Deutsche zu übertragen. In: Schmid, Herta (Hg.): Kapitel zur Poetik Karel Hynek Máchas. München, Sagner. S. 133-145.

Jakobson, Roman (1995): K popisu Máchova verše. In: Jakobson, Roman: Poetická funkce. Jinočany, Nakl. H \& H. S. 427-476.

Janský, Karel (1958): Karel Hynek Mácha ve vzpominkách současníkư. Praha, Svobodné Slovo, Melantrich.

Kaiser, Max / Michler, Werner (2002): Das literarische Feld und das Terrain der Politik. In: Böhler, Michael (Hg.): Kulturtopographie deutschsprachiger Literaturen. Tübingen, Niemeyer. S. 179-230.

Kapper, Siegfried (1842): Karel Hynek Macha [sic] und die neuböhmische Literatur. In: Sonntagsblätter 18/1842. S. 313-314.

Králík, Oldřich (1961): Zu den deutschen Gedichten Karel Hynek Máchas. In: Zeitschrift für Slawistik 6/3/1961. S. 387-414.

Králík, Oldřich (1962): Zu den deutschen Gedichten Karel Hynek Máchas. In: Zeitschrift für Slawistik 7/1/1962. S. 60-101.

Krčma, František (1932a): Starší německé studie o K. H. Máchovi. In: Listy filologické 4/1932. S. 404-413.

Krčma, František (1932b): K. H. Mácha. Soupis praci o jeho životě, dile a kultu. Praha, Svaz knihkupcův a nakladatelů ČSR.

Křen, Jan (1990): Konfliktni společenstvi. Češi a Němci 1780-1918. Praha, Academia.

Mácha, Karel Hynek (1844): Karel Hynek Mácha’s Mai. Übers. Kapper, S. In: Libussa. Jahrbuch für 1844. 3/1844. S. 97-124.

Mácha, Karel Hynek (1862): Karl Hynek Mácha’s ausgewählte Gedichte. Übers. Waldau, Alfred. Prag, Verlag H. Dominicus. S. 42-86.

Mácha, Karel Hynek (1882): Mai. Lyrisch-romantisches Gedicht. Übers. Müller, Karl Leitmeritz, Verlag von Wirth u. Comp.

Mácha, Karel Hynek (1959): Básně a dramatické zlomky. Hrsg. v. Karel Janský. Praha, SNKLU.

Mácha, Karel Hynek (1972): Literárni zápisniky, deniky, dopisy. Hrsg. v. Karel Janský. Praha, SNKLU.

Mácha, Karel Hynek (2000): Die Liebe ging mit mir... Stuttgart / München, Dt. VerlagsAnstalt.

Mácha, Karel Hynek (2002): Básně. Hrsg. v. Miroslav Červenka. Praha, Nakladatelství Lidové noviny.

Mácha, Karel Hynek (2006): Máj. Mai. Hrsg. v. Pavel Vašák. Praha, Akropolis.

Mácha, Karel Hynek (2008): Prózy. Hrsg. v. Zdeněk Hrbata / Martin Procházka. Praha, Lidové noviny.

Mácha, Karel Hynek (2012): Máj. Mai. Übers. Cikán, Ondřej. Wien, Labor.

Mácha, Karel Hynek / Rambousek, Bernard Alois (2010): Máj. Der Mai. Hrsg. v. Lukáš Novosad / Miloslav Uličný / Astrid Winter. Praha, Splav.

Mukařovský, Jan (1948): Máchovské studie. Praha, Nakladatelství Svoboda.

Nezdařil, Ladislav (1985): Česká poezie v némeckých překladech. Praha, Academia.

Peřina, Josef (1996): Přehledné dějiny vztahù české a německo-české literatury v 19. století. Sv. I (1780-1848). Ústí n. Labem, Albis international.

Polák, Karel (1940): Německé překlady Máje. In: Hartl, Antonín et al. (Hg.): Věčný Mácha. Praha, Čin. S. 215-238.

Přibil, Marek (2009): Mácha a německý romantismus. In: Svět literatury 40/2009. S. 16-28. 
Prokop, Dušan (2010): Kniha o Máchově Máji. Praha, Academia.

Puchmajer, Antonín Jaroslav (1833): Fialky. Praha, Kober.

Schamschula, Walter (1983): Gedanken beim Übersetzen des Máj. In: Mácha, Karel Hynek: Máj. Zweisprachige Ausgabe. Übers. Babler, Otto F. / Schamschula, Walter. Köln, Böhlau. S. 119-126.

Schulze, Ernst (1818): Die bezauberte Rose. Leipzig, Brockhaus.

Striedter, Jurij (1963): K. H. Mácha als Dichter der europäischen Romantik. In: Zeitschrift für Slavische Philologie 31/1963. S. 42-90.

Tschižewskij, Dmitrij I. (1972): Zu Máchas Weltanschauung. In: Tschižewskij, Dmitrij I.: Kleinere Schriften. Bd. II. München, Fink. S. 240-286.

Tureček, Dalibor (2010): KHM 1810-2010: Dvě století české kultury s Máchou. Praha, Památník národního písemnictví.

Uličný, Miloslav (2010a): Vedlejší efekt. Mácha’s Mai. In: Plav 1/2010. S. 49-54.

Uličný, Miloslav (2010b): Nezvěstný Bernard Rambousek. In: Mácha, Karel Hynek / Rambousek, Bernard Alois: Máj. Der Mai. Hrsg. v. Lukáš Novosad / Miloslav Uličný / Astrid Winter. Praha, Splav. S. 9-34.

Vašák, Pavel (1978): Neznámá recenze Máje z Unser Planet 1836. In: Česká literatura 26/3/1978. S. 248-259.

Vašák, Pavel (2004): Literárni pout Karla Hynka Máchy. Praha, Academie.

Weinolt, Franz (1835): Denkwürdigkeiten aus dem Leben Alois Klar'. Prag / Leitmeritz, [s. n.].

Winter, Aastrid (2010): Doslov. In: Mácha, Karel Hynek / Rambousek, Bernard Alois: Máj. Der Mai. Hrsg. v. Lukáš Novosad / Miloslav Uličný / Astrid Winter. Praha, Splav. S. $102-118$.

Winter, Astrid (2011): Between the East and West. The German Reception of Mácha’s Máj in the $19^{\text {th }}$ Century. In: Translatologica Pragensia 8/2011. S. 188-199.

Winter, Astrid (2013): „Unnahmlich und unübersetzlich“. Die deutsche Mácha-Rezeption im 19. Jahrhundert am Beispiel der Máj-Übersetzungen. In: Deutsche Beiträge zum 15. Internationalen Slavistenkongress, Minsk 2013. Hrsg. v. Sebastian Kempgen / Monika Wingender / Norbert Franz / Miranda Jakiša. München [u. a.], Sagner. S. 421-433.

\author{
Astrid Winter \\ Ústav translatologie \\ Filozofická fakulta \\ Univerzity Karlovy v Praze \\ Hybernská 3 \\ 11000 Praha 1 \\ winterastrid@web.de
}

